\title{
Association between Helicobacter pylori Infection and Colorectal Adenomatous Polyps
}

\author{
Wen Yang $\mathbb{D}^{1,2}$ and Xueqing Yang ${ }^{3}$ \\ ${ }^{1}$ No. 6 Medical Center of the PLA General Hospital, No. 6 Fuchenglu Road, Beijing 100048, China \\ ${ }^{2}$ Department of Gastroenterology and Hepatolog, Sunshine Union Hospital, Weifang, Shandong Province 261061, China \\ ${ }^{3}$ Economic Department, University of Miami, 5250 University Dr. Coral Gables, FL 33146, USA
}

Correspondence should be addressed to Wen Yang; yangwen666123@sohu.com

Received 9 September 2019; Accepted 27 November 2019; Published 18 December 2019

Academic Editor: Kazuhiko Uchiyama

Copyright (c) 2019 Wen Yang and Xueqing Yang. This is an open access article distributed under the Creative Commons Attribution License, which permits unrestricted use, distribution, and reproduction in any medium, provided the original work is properly cited.

\begin{abstract}
Background. Helicobacter pylori infection is a common chronic infection worldwide. At the same time, the incidence of colorectal adenomatous polyps is also at high levels. In order to assess the relationship between Helicobacter pylori infection and the occurrence of colorectal adenomatous polyps, we observed 166 patients who had undergone an electronic colonoscopy and ${ }^{13} \mathrm{C}$ urea breath test in the outpatient clinic. Method. A total of 166 (87 males and 79 females, aged $53.85 \pm 9.18$ years) patients who had colonoscopy examination and ${ }^{13} \mathrm{C}$ urea breath test were divided into a Helicobacter pylori-positive group $(n=68)$ and Helicobacter pylori-negative group $(n=98)$ by the ${ }^{13} \mathrm{C}$ urea breath test. At the same time, total cholesterol, triglycerides, and fasting blood sugar were measured and the occurrence of hypertension was counted. Results. Patients with Helicobacter pylori infection had higher incidence of colorectal adenomatous polyps and multiple colorectal adenomatous polyps, higher levels of total cholesterol and fasting glucose, and more males $(P<0.05, P<0.01)$. It was found that Helicobacter pylori infection $(P<0.05$, OR 2.383) was significantly associated with the risk of colorectal adenomatous polyps by binary logistic regression analysis. Conclusions. Patients with Helicobacter pylori infection had higher incidence of colorectal adenomatous polyps.
\end{abstract}

\section{Introduction}

Helicobacter pylori is a common gram-negative bacterium that dwells on the gastric mucosa and can secrete urea enzymes, vacuolus toxins, and cytotoxin-related genes. Studies have shown that the prevalence of Helicobacter pylori infection in the general population is more than $50 \%$ [1]. It is recognized as the culprit of chronic gastritis, gastric ulcers, and gastric cancer. In addition to causing the gastrointestinal disease, it is also thought to have extragastrointestinal effects, including idiopathic thrombocytopenia purpura, chronic idiopathic urticaria, iron deficiency anemia, arteriosclerosis, and ischemic heart disease [2-4].

In recent years, several studies demonstrated that Helicobacter pylori infection is involved in metabolic syndrome which is composed of the following major components: abdominal obesity, insulin resistance (IR), elevated BP, and dyslipidemia [5].
The colorectal adenomatous polyp is a common disease of the digestive system and is closely related to the occurrence of colorectal cancer. Studies have confirmed that metabolic syndrome is a risk factor for colorectal cancer [6]. To further explore the relationship between Helicobacter pylori infection and colorectal adenomatous polyps, we observed the relationship between Helicobacter pylori infection and colorectal adenomatous polyps by means of electronic colonoscopy.

\section{Material and Method}

2.1. Study Population and Data Collection. This study was conducted at Sunshine Union Hospital. We followed the methods of Yang et al. [7]. All the qualified subjects who took part in the study were hospitalized from January 2019 to July 2019. Inclusion criteria were complete, available medical records and having gastroscopy in our hospital; having not used proton pump inhibitors (PPIs), histamine type 
TABLE 1: Comparison of the variables of patients in the healthy control and Helicobacter pylori infection group.

\begin{tabular}{lccc}
\hline Variables & Healthy control $(n=98)$ & Helicobacter pylori infection $(n=68)$ & $P$ value \\
\hline Mal, $n(\%)$ & $42(62.86)$ & $45(66.18)$ & 0.003 \\
Age & $53.551 \pm 9.276$ & $54.279 \pm 9.098$ & $36(52.94)$ \\
Colorectal adenomatous polyps, $n(\%)$ & $30(30.61)$ & $27(39.70)$ & 0.617 \\
Multiple colorectal adenomatous polyps, $n(\%)$ & $13(13.26)$ & $7(10.29)$ & 0.004 \\
Single colorectal adenomatous polyp, $n(\%)$ & $8(8.16)$ & $5.152 \pm 1.054$ & 0.001 \\
Total cholesterol (mmol/L) & $4.564 \pm 1.586$ & $1.833 \pm 1.625$ \\
Triglycerides (mmol/L) & $1.477 \pm 1.131$ & $13(19.12)$ & 0.008 \\
Hypertension, $n(\%)$ & $12(12.24)$ & $5.593 \pm 1.417$ \\
Fasting glucose $(\mathrm{mmol} / \mathrm{L})$ & $4.978 \pm 1.796$ & 0.098 \\
\hline
\end{tabular}

2 receptor antagonists $(\mathrm{H} 2 \mathrm{~A})$, antibiotics, bismuth, or sucralfate for up to one month prior to the endoscopy; normal blood pressure (diastolic blood pressure (DBP) of less than $90 \mathrm{mmHg}$ and systolic blood pressure (SBP) of less than $150 \mathrm{mmHg}$ ); or well-controlled hypertension (DBP of less than $90 \mathrm{mmHg}$ and SBP of less than $150 \mathrm{mmHg}$ ). Exclusion criteria were the presence of hematological diseases; past history of cancer; major gastrointestinal surgery, including partial or total gastrectomy or colectomy; pulmonary disease; diabetes mellitus; smoking; alcohol consumption; kidney diseases; pregnancy and lactation; and having been using medicine which is known to be able to effect blood coagulation, such as aspirin and clopidogrel. Written informed consent was obtained from each subject and was recorded by the physician who explained the study procedures. The study was reviewed and approved by the Ethics and Research Committee of Sunshine Union Hospital (Shandong, China), and the reported investigations were carried out in accordance with the principles of the Declaration of Helsinki as revised in 2000 .

2.1.1. Diagnosis of Colorectal Polyps. The operations of colonoscopy were performed by physicians who had been trained for 6 months or longer at our hospital and had carried out 500 or more colonoscopies. Insertion up to the cecum was successful in $96.8 \%$ of examinations. Anal and rectal finger examination was the routine prior to insertion of the endoscope. After arriving at the end of the ileum, slowly exit the colonoscopy and carefully observe the colorectal wall mucosa, and retreat time is not less than 6 minutes. In preparation for colonoscopy, subjects took a laxative (polyethylene glycol electrolyte powder, Beaufour Ipsen Industrie, Dreux, France) on the night before the examination and on the day of colonoscopy at $08: 30 \mathrm{~h}$ (colonic lavage using approximately $2000 \mathrm{~mL}$ of solution was carried out). Smallcaliber electronic colonoscopies (CF-200I, 240I, 240AI, and Q260AI; Olympus, Tokyo, Japan) were used for colonoscopy. All visualized lesions were biopsied and histologically assessed by experienced pathologists.

2.2. Helicobacter pylori Infection Test. The diagnosis of Helicobacter pylori infection was based on the results of the fasting ${ }^{13} \mathrm{C}$ urea breath test $\left({ }^{13} \mathrm{C}\right.$-UBT $)$. Breath tests were conducted by dedicated nurses. Subjects fasted overnight and maintained normal exhalation. A straw was inserted into the bottom of a sample tube. The straw was slowly inhaled into the sample tube for 4 to 5 seconds, and the straw was pulled out. Immediately tighten the test tube cover. This method collects 0 minute of exhalation. The patient took a bottle of urea ${ }^{13} \mathrm{C}$ particles with 80 to $100 \mathrm{~mL}$ cold drinking water and sat still. According to the above method of collecting exhalation, the patient collected 30 minutes of exhalation after taking urea ${ }^{13} \mathrm{C}$ and twisted the test tube cover. The 0 -minute and 30-minute exhalation sample tubes collected will be tested on the corresponding instrument for ${ }^{13} \mathrm{C} \mathrm{O}_{2}$ (the reagent kit will be provided by Beijing Buzhan Pharmaceutical Co., Ltd., and the instrument will be provided by Beijing Huayuan Anbang Technology Co., Ltd-type ${ }^{13} \mathrm{C}$ infrared spectrometer). The ${ }^{13} \mathrm{C}$ urea exhalation test was positive for Helicobacter pylori infection as the delta over baseline is greater than or equal to 4.0 , and the ${ }^{13} \mathrm{C}$ urea exhalation test was negative for Helicobacter pylori infection as the delta over baseline is less than 4.0.

2.3. Biochemical Analyses. Blood samples were taken into anticoagulated tubes from participants after an overnight fast of more than $12 \mathrm{~h}$. Plasma was separated by centrifugation at $3000 \times g$ for 10 minutes at room temperature. The levels of creatinine, BUN, total cholesterol, triglyceride, and glucose were measured using a multichannel analyzer (Roche Hitachi 737; Boehringer Mannheim Diagnostics, USA).

2.4. Statistical Analysis. Data were expressed as mean \pm SD or counts. Statistical analysis was performed using SPSS version 16.0 (SPSS Inc., Chicago, IL), and the level of statistical significance was defined as $P<0.05$. The independent samples $t$-test was used for the comparisons of continuous data, while the chi-square test was used for the comparisons of categorical variables. Binary logistic regression analysis was used to determine the factors that were associated with colorectal adenomatous polyps.

\section{Results}

3.1. Comparison of Variables in the Healthy Control and the Helicobacter pylori Infection Group. Comparisons of the variables between the healthy control and the Helicobacter pylori infection group are shown in Table 1. Patients with 
TABLE 2: The results of binary logistic regression analysis of colorectal adenomatous polyps.

\begin{tabular}{|c|c|c|c|c|c|c|c|}
\hline & & \multirow{2}{*}{ Beta } & \multirow{2}{*}{ SE } & \multirow{2}{*}{$P$ value } & \multirow{2}{*}{ OR } & \multicolumn{2}{|c|}{$95 \% \mathrm{CI}$} \\
\hline & & & & & & Lower & Upper \\
\hline Mal, $n$ & Female $=0$, male $=1$ & 0.278 & 0.342 & 0.417 & 1.321 & 0.675 & 2.583 \\
\hline Age, years & $<60=0, \geq 60=1$ & 0.084 & 0.382 & 0.826 & 1.088 & 0.514 & 2.301 \\
\hline Helicobacter pylori infection, $n$ & Absent $=0$, present $=1$ & 0.868 & 0.344 & 0.012 & 2.383 & 1.214 & 4.676 \\
\hline Total cholesterol (mmol/L) & $<5.69=0, \geq 5.69=1$ & 0.140 & 0.359 & 0.696 & 1.151 & 0.569 & 2.327 \\
\hline Triglycerides $(\mathrm{mmol} / \mathrm{L})$ & $<1.7=0, \geq 1.7=1$ & -0.209 & 0.373 & 0.575 & 0.811 & 0.391 & 1.685 \\
\hline Hypertension, $n$ & Absent $=0$, present $=1$ & 0.277 & 0.481 & 0.564 & 1.319 & 0.514 & 3.385 \\
\hline Fasting glucose (mmol/L) & $<6.1=0, \geq 6.1=1$ & -0.092 & 0.454 & 0.839 & 0.912 & 0.375 & 2.220 \\
\hline
\end{tabular}

Helicobacter pylori infection had higher incidence of colorectal adenomatous polyps and multiple colorectal adenomatous polyps, higher levels of total cholesterol and fasting glucose, and more males $(P<0.05, P<0.01)$.

3.2. Helicobacter pylori Infection and Risk Factors for Colorectal Adenomatous Polyps. Binary logistic regression analysis was used to evaluate the risk factors for colorectal adenomatous polyps. Colorectal adenomatous polyps were taken as the dependent variable, and age, gender, Helicobacter pylori infection, total cholesterol, triglyceride, fasting glucose, and hypertension were taken as independent variables. It was found that Helicobacter pylori infection $(P<0.05$, OR 2.383) was significantly associated with the risk of colorectal adenomatous polyps (Table 2).

\section{Discussion}

Colorectal adenomatous polyps can be considered precancerous lesions, for hyperproliferative epithelial cells can transform from the adenomatous stage to the colorectal cancer stage $[8,9]$. Early diagnosis of adenomatous polyps depends on colonoscopy. With the popularity of colonoscopy, colorectal adenomatous polyps were discovered early and removed in time. The pathogenesis of colorectal adenomatous polyps remains unclear. From the results of this study, it is found that Helicobacter pylori infection is related to colorectal adenomatous polyps, which is in agreement with the previous studies $[10,11]$.

The exact mechanism of Helicobacter pylori infection and colorectal adenomatous polyps is not yet clear. The following mechanisms may help explain our findings. First, Helicobacter pylori infection could upregulate the expression of matrix metalloproteinases [12], which may take part in not only colorectal carcinogenesis from adenomatous polyps but also colorectal tumor invasion and initiation of a metastatic cascade [13]. Second, Helicobacter pylori infection can lead to abnormal secretion of ghrelin secreted by the gastric mucosa [14], and ghrelin can prevent the occurrence of colorectal adenomatous polyps and colorectal cancer [15]. Third, Helicobacter pylori effects the inflammatory state of the intestine through the release of cytotoxins, lipopolysaccharide (LPS), and other toxic substances [16], which are involved in the formation of colorectal adenomatous polyps [17].
In short, from the results of this study, we can see that Helicobacter pylori infection is associated with the occurrence of colorectal adenomatous polyps, so the treatment of Helicobacter pylori infection may help prevent colorectal adenomatous polyps.

The shortcomings of this study include the following: first, the number of sample cases observed is small; secondly, the subjects selected for observation are those who go to the hospital for medical treatment or health examination. Furthermore, there was no in-depth study on the internal mechanism of increased incidence of Helicobacter pylori infection and colorectal adenomatous polyps.

\section{Data Availability}

The data used to support the findings of this study are available from the corresponding author upon request.

\section{Disclosure}

The funding organizations had no role in the design and concept of the study; the collection, management, analysis, and interpretation of the data; or the preparation, review, or approval of the manuscript.

\section{Conflicts of Interest}

There are no conflicts of interest that are directly relevant to the content of this study.

\section{Authors' Contributions}

Yang Wen researched and analyzed the data and wrote the manuscript. Yang Xueqing analyzed the data and wrote the manuscript. All authors reviewed the manuscript, approved the final draft, and decided to submit it for publication.

\section{Acknowledgments}

This work was supported by the Special Topics of Health Care for the Chinese People's Liberation Army (17BJZ03). 


\section{References}

[1] S. Suerbaum and P. Michetti, "Helicobacter pylori infection," The New England Journal of Medicine, vol. 347, no. 15, pp. 1175-1186, 2002.

[2] S. F. Ameriso, E. A. Fridman, R. C. Leiguarda, and G. E. Sevlever, "Detection of Helicobacter pylori in human carotid atherosclerotic plaques," Stroke, vol. 32, no. 2, pp. 385-391, 2001.

[3] A. K. Adiloglu, C. Nazli, B. Cicioglu-Aridogan, O. Kinay, R. Can, and O. Ergene, "Gastroduodenal Helicobacter pylori infection diagnosed by Helicobacter pylori stool antigen is related to atherosclerosis," Acta Cardiologica, vol. 58, no. 4, pp. 335-339, 2003.

[4] M. A. Mendall, P. M. Goggin, N. Molineaux et al., "Relation of Helicobacter pylori infection and coronary heart disease," British Heart Journal, vol. 71, no. 5, pp. 437-439, 1994.

[5] Y. Y. Chen, W. H. Fang, C. C. Wang et al., "Helicobacter pylori infection increases risk of incident metabolic syndrome and diabetes: a cohort study," PLoS One, vol. 14, no. 2, article e0208913, 2019

[6] Q. He, H. Zhang, S. Yao et al., "A study on relationship between metabolic syndrome and colorectal cancer," Journal of BUON, vol. 23, no. 5, pp. 1362-1368, 2018.

[7] W. Yang and C. Xuan, "Influence of _Helicobacter pylori_ Infection on Metabolic Syndrome in Old Chinese People," Gastroenterology Research and Practice, vol. 2016, Article ID 6951264, 4 pages, 2016.

[8] L. Maggio-Price, P. Treuting, W. Zeng, M. Tsang, H. Bielefeldt-Ohmann, and B. M. Iritani, "Helicobacter infection is required for inflammation and colon cancer in Smad3-deficient mice," Cancer Research, vol. 66, no. 2, pp. 828-838, 2006.

[9] A. Soylu, S. Ozkara, H. Alıs et al., "Immunohistochemical testing for Helicobacter pylori existence in neoplasms of the colon," BMC Gastroenterology, vol. 8, no. 1, pp. 1-6, 2008.

[10] F. Teimoorian, M. Ranaei, K. Hajian Tilaki, J. Shokri Shirvani, and Z. Vosough, "Association of Helicobacter pylori infection with colon cancer and adenomatous polyps," Iranian Journal of Pathology, vol. 13, no. 3, pp. 325-332, 2018.

[11] J. Kountouras, N. Kapetanakis, C. Zavos, and I. Romiopoulos, "Impact of Helicobacter pylori infection on normal colorectal mucosa, adenomatous polyps and adenocarcinoma sequence," Colorectal Disease, vol. 16, no. 5, pp. 390-391, 2014.

[12] Y. C. Yeh, B. S. Sheu, H. C. Cheng, Y. L. Wang, H. B. Yang, and J. J. Wu, "Elevated serum matrix metalloproteinase- 3 and -7 in $\mathrm{H}$. pylori-related gastric cancer can be biomarkers correlating with a poor survival," Digestive Diseases and Sciences, vol. 55, no. 6, article 926, pp. 1649-1657, 2010.

[13] M. Groblewska, B. Mroczko, M. Gryko, B. Kędra, and M. Szmitkowski, "Matrix metalloproteinase 2 and tissue inhibitor of matrix metalloproteinases 2 in the diagnosis of colorectal adenoma and cancer patients," Folia Histochemica et Cytobiologica, vol. 48, no. 4, pp. 564-571, 2010.

[14] C. Romo-González, E. Mendoza, R. M. Mera et al., "Helicobacter pylori infection and serum leptin, obestatin, and ghrelin levels in Mexican schoolchildren," Pediatric Research, vol. 82, no. 4, pp. 607-613, 2017.

[15] G. Murphy, A. J. Cross, S. M. Dawsey et al., "Serum ghrelin is associated with risk of colorectal adenocarcinomas in the ATBC study," Gut, vol. 67, no. 9, pp. 1646-1651, 2018.
[16] S. Yokota, T. Ohnishi, M. Muroi, K. Tanamoto, N. Fujii, and K. Amano, "Highly-purified Helicobacter pylori LPS preparations induce weak inflammatory reactions and utilize Toll-like receptor 2 complex but not Toll-like receptor 4 complex," FEMS Immunology and Medical Microbiology, vol. 51, no. 1, pp. 140-148, 2007.

[17] Q. Guo, S. Shen, X. Li, K. Tang, and W. Zhou, "Inflammatory factors promote the development of colorectal cancer," Zhong Nan Da Xue Xue Bao Yi Xue Ban, vol. 36, no. 7, pp. 646-649, 2011. 


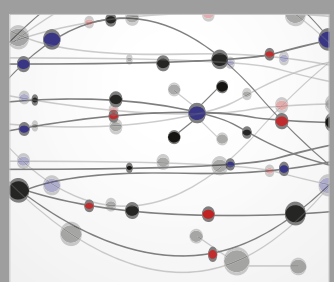

The Scientific World Journal
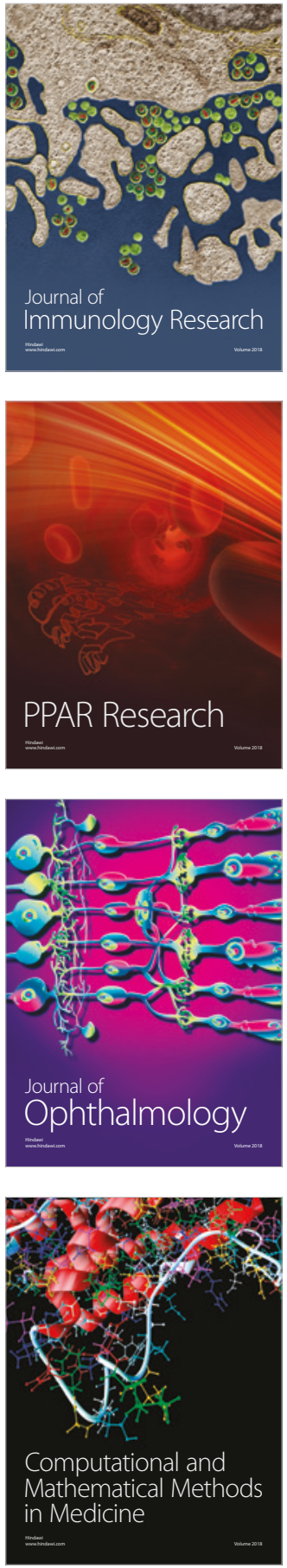

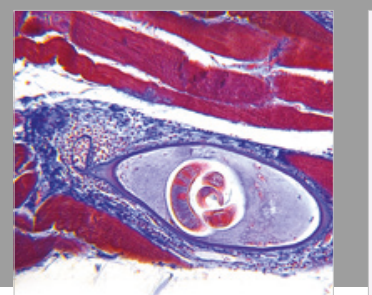

Gastroenterology Research and Practice

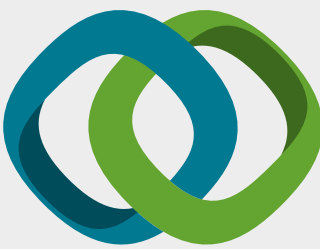

\section{Hindawi}

Submit your manuscripts at

www.hindawi.com
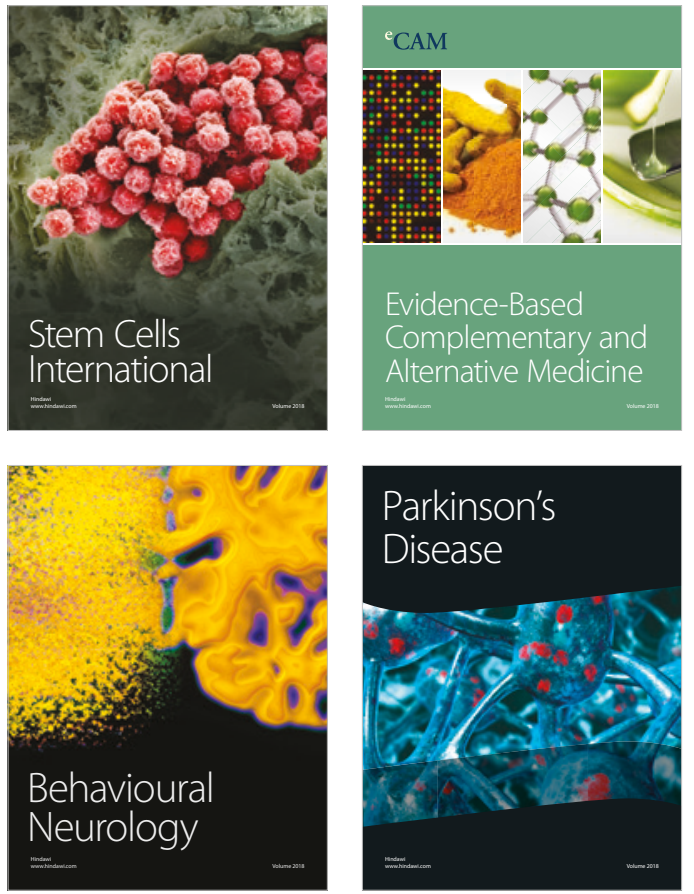

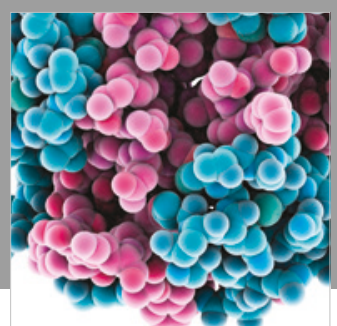

ournal of

Diabetes Research

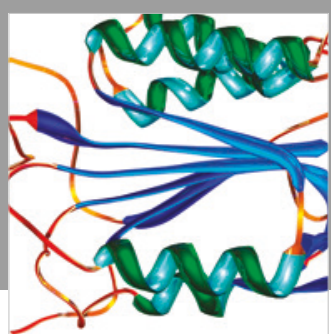

Disease Markers
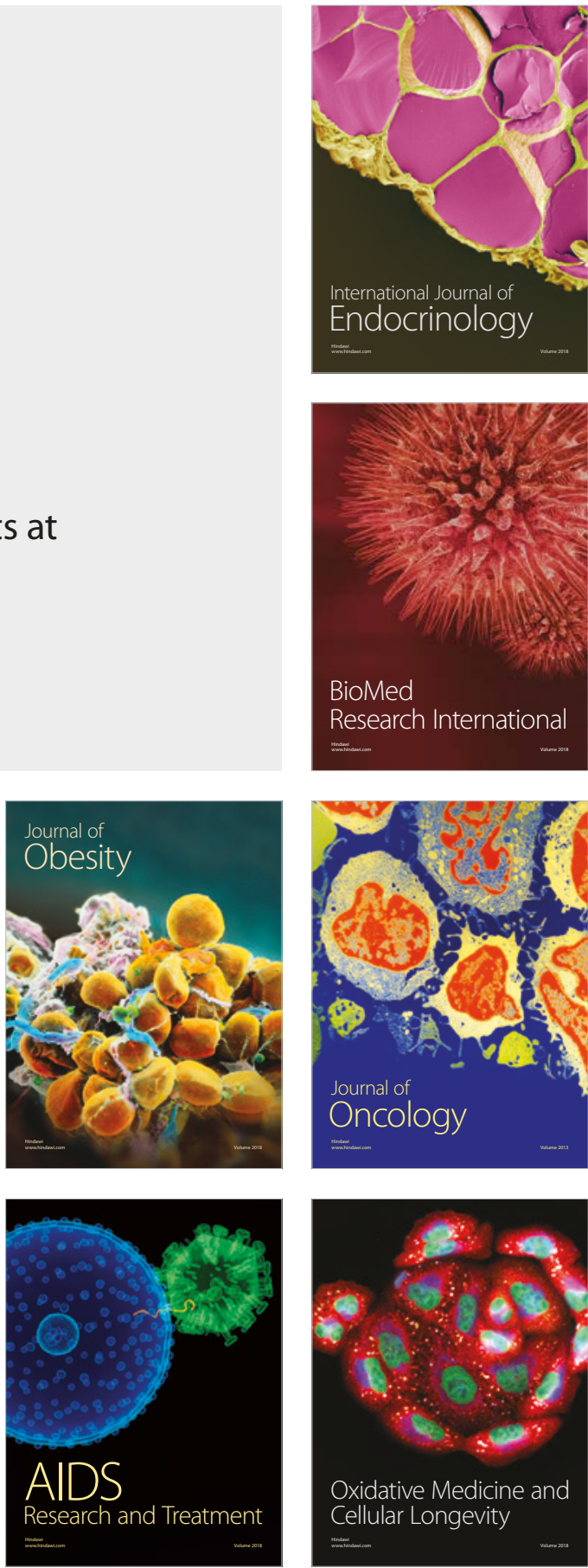